\title{
Forward scattering from square cylinders in the resonance region with application to aperture blockage
}

Rusch, W.; Appel-Hansen, Jørgen; Klein, C; Mittra, R

Published in:

I E E E Transactions on Antennas and Propagation

Publication date:

1976

Document Version

Publisher's PDF, also known as Version of record

Link back to DTU Orbit

Citation (APA):

Rusch, W., Appel-Hansen, J., Klein, C., \& Mittra, R. (1976). Forward scattering from square cylinders in the resonance region with application to aperture blockage. I E E E Transactions on Antennas and Propagation, 24(2), 182-189.

\section{General rights}

Copyright and moral rights for the publications made accessible in the public portal are retained by the authors and/or other copyright owners and it is a condition of accessing publications that users recognise and abide by the legal requirements associated with these rights.

- Users may download and print one copy of any publication from the public portal for the purpose of private study or research.

- You may not further distribute the material or use it for any profit-making activity or commercial gain

- You may freely distribute the URL identifying the publication in the public portal 


\title{
Forward Scattering from Square Cylinders in the Resonance Region with Application to Aperture Blockage
}

\author{
W. V. T. RUSCH, SENIOR MEMBER, IEEE, JØRGEN APPEL-HANSEN, MEMBER, IEEE, CHARLES A. KLEIN, \\ AND RAJ MITTRA, FELLOW, IEEE
}

\begin{abstract}
The relationship between the induced field ratio (TFR) of a cylinder and aperture blocking of a constant-phase aperture by cylindrical struts is discussed. An analytical technique is presented whereby the IFR of rectangular cylinders can be calculated using the method-of-moments with internal constraint points. An experimental technique using a forward-scattering range is used to measure the IFR's of square and circular cylinders in an anechoic chamber. These experimental results are compared with the theory, and their implications on aperture blocking losses and boresight cross polarization are discussed.
\end{abstract}

\section{INTRODUCTION}

$\mathrm{T}$ HE PRESENCE of long thin mechanical support structures in front of a constant-phase aperture, such as that of a focused paraboloid, has generally defied rigorous attempts to analyze their effects upon the RF performance of the aperture. However, as a good first approximation, it has been proposed [1]-[4] that the strut currents are the same currents that would flow on an infinite cylindrical structure of the same cross section in free-space immersed in an infinite linearly polarized plane wave with the same polarization and direction of incidence as the local geometrical ray incident upon that part of the strut as it emerges from the aperture (transmit mode). This approximation is known as the "IFR-hypothesis."

When an infinite cylinder is immersed in an incident plane wave (Fig. 1), its induced field ratio (IFR) is defined as the ratio of the forward-scattered field to the hypothetical field radiated in the forward direction by the plane wave in the reference aperture of width equal to the shadow of the geometrical cross section of the cylinder on the incident wavefront [1]. Thus for the $E$-vector of the incident plane wave parallel to the cylinder axis

$$
\mathrm{IFR}_{E}=-\frac{Z_{0}}{2\left(\xi_{2}-\xi_{1}\right) E_{0}} \int_{S_{1}} J_{S z} e^{j k \rho^{\prime} \sin \phi^{\prime}} d l
$$

where $E_{0}$ is the electric intensity of the plane wave, $Z_{0}$ is the intrinsic impedance of free space, $J_{S z}$ is the axial component of surface-current density, and $S_{1}$ is the line

Manuscript received January 27, 1975.

W. V. T. Rusch is with the Department of Electrical Engineering, University of Southern California, Los Angeles, CA 90007. During this work he was with the Electromagnetics Institute, Technical University of Denmark, Lyngby, Denmark.

J. Appel-Hansen is with the Electromagnetics Institute, Technical University of Denmark, Lyngby, Denmark.

C. A. Klein is with Aeronutronic-Ford, Palo Alto, CA.

R. Mittra is with the Electromagnetics Laboratory, University of Illinois, Urbana, IL 61801.

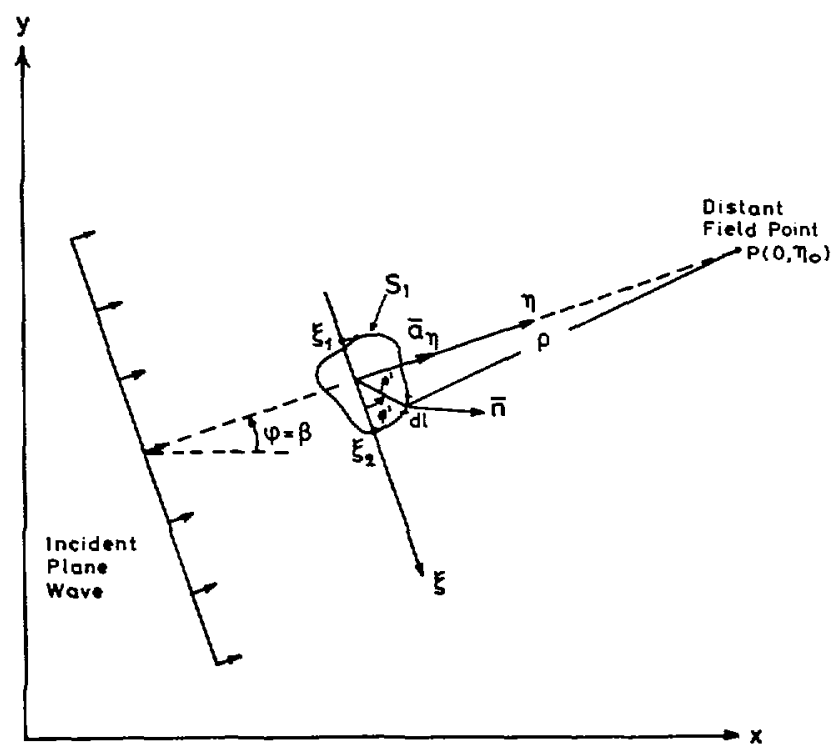

Fig. 1. IFR geometry.

contour defining the cylinder's periphery. Similarly, for the $H$-vector parallel to the cylinder axis

$$
\operatorname{IFR}_{H}=\frac{1}{2\left(\xi_{2}-\xi_{1}\right) H_{0}} \int_{S_{1}} H_{z}\left(a_{\eta} \cdot n\right) e^{j k \rho^{\prime} \sin \phi^{\prime}} d l
$$

where $H_{0}$ is the magnetic field of the incident wave, $H_{z}$ is the total magnetic field in the axial direction at the surface of the cylinder, and the unit vectors $a_{\eta}$ and $n$ are defined in the figure. The IFR $E$ and IFR ${ }_{H}$ for a right-circular cylinder of radius $a$ are given by [2]

$$
\operatorname{IFR}_{E}=-\frac{1}{k a \cos \alpha} \sum_{n=-\infty}^{+\infty} J_{n}(k a \cos \alpha) / H_{n}{ }^{(2)}(k a \cos \alpha)
$$

$\mathrm{IFR}_{H}=-\frac{1}{k a \cos \alpha} \sum_{n=-\infty}^{+\infty} J_{n}{ }^{\prime}(k a \cos \alpha) / H_{n}^{(2)^{\prime}}(k a \cos \alpha)$

where $J_{n}$ and $H_{n}^{(2)}$ are, respectively, the Bessel and outgoing Hankel function, and the prime indicates the derivative with respect to the argument. These formulas have also included the effects of a tilt angle $\alpha$ between the incident wavefront and the cylinder axis. Thus for the nonnormal incidence the IFR is determined for an equivalent cylinder 


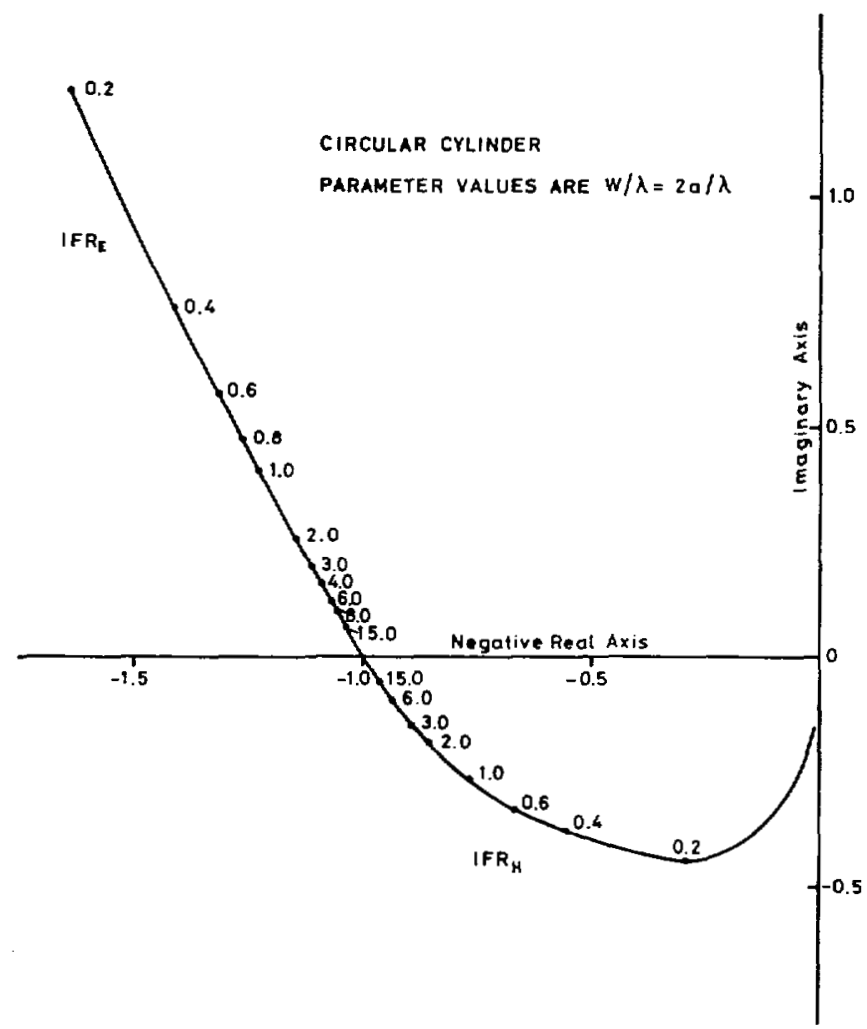

Fig. 2. Complex $\mathrm{IFR}_{E}$ and $\mathrm{IFR}_{B}$ for circular cylinder.

with linear dimensions of the cross section reduced by the factor $\cos (\alpha)$. This principle applies equally well to other cross sections in general. The IFR's for the right-circular cylinder are plotted on the complex plane in Fig. 2. In general, the $\mathrm{IFR}_{E}$ is larger in magnitude than the $\mathrm{IFR}_{H}$ and has a positive phase angle compared to a negative phase angle for the $H$ polarization. Both IFR's approach the value $-1.0+j 0.0$ as the radius increases, one from below and the other from above.

Using the IFR-hypothesis in a practical application, the loss in boresight gain due to central blockage and the planewave component of strut current for a linearly polarized aperture is

\section{LOSS (dB)}

$$
\begin{aligned}
& =-20 \log _{10} \mid 1-\frac{\int_{0}^{2 \pi} \int_{0}^{\beta} F(t, \phi) t d t d \phi}{\int_{0}^{2 \pi} \int_{0}^{1} F(t, \phi) t d t d \phi} \\
& +\sum_{i=1}^{\substack{\text { number } \\
\text { struts }}}\left\{\left(w_{i} \frac{D}{2}\right)\left(\mathrm{IFR}_{E i}^{\prime} \cos ^{2} \gamma_{i}+\mathrm{IFR}_{H i} \sin ^{2} \gamma_{i}\right)\right. \\
& \\
& +\frac{\int_{\beta}^{\beta_{i}} F\left(t, \phi_{i}\right) d t}{\left.\left(\frac{D}{2}\right)^{2} \int_{0}^{2 \pi} \int_{0}^{1} F(t, \phi) t d t d \phi\right) \mid}
\end{aligned}
$$

where

$\begin{array}{ll}t & \text { normalized radial aperture coordinate, } \\ \phi & \text { azimuthal aperture coordinate, } \\ F(t, \phi) & \text { aperture distribution function, } \\ \beta & \text { fractional diameter blocking by central blockage, } \\ w_{i} & \text { width of } i \text { th strut, } \\ D & \text { aperture diameter, } \\ \beta_{i} & \text { fractional radius blocking by } i \text { th strut, } \\ \gamma_{i} & \text { angle between electric vector and } i \text { th strut, } \\ \alpha_{i} & \text { angle between ith strut and aperture plane, } \\ \mathrm{IFR}_{E i}{ }^{\prime} & \mathrm{IFR}_{E} \text { for } i \text { th strut cross section with linear } \\ \mathrm{IFR}_{H i}{ }^{\prime} & \mathrm{IFR}_{H} \text { for } i \text { th strut cross section with linear } \\ & \text { dimensions reduced by } \cos \alpha_{i} .\end{array}$

The second term represents the "optical" approximation to axially symmetric blockage. The third term represents a summation over the plane-wave component of strut currents on each strut. For a uniformly illuminated aperture, (3) reduces to

$$
\begin{aligned}
& \text { LOSS (dB) } \\
& =-20 \log _{10} \mid 1-\beta^{2}+\sum_{i}\left\{\frac { A _ { i } } { ( \pi \frac { D } { 2 } ) ^ { 2 } } \left(\mathrm{IFR}_{E i}{ }^{\prime} \cos ^{2} \gamma_{i}\right.\right. \\
& \left.\left.+\operatorname{IFR}_{H i} \sin ^{2} \gamma_{i}\right)\right\}
\end{aligned}
$$

where $A_{i}$ is the projected area of the $i$ th strut on the aperture plane.

In addition to degrading boresight gain, a feed-support strut will generate boresight cross polarization if it is not aligned parallel to or perpendicular to the electric field in the aperture. The boresight cross polarization level due to the plane-wave component of strut currents is

$$
\begin{aligned}
& \mathrm{CP}(\mathrm{dB}) \\
& =20 \log _{10} \mid \sum_{i}\left\{\left(w_{i} \frac{D}{2}\right)\left(\mathrm{IFR}_{H i}{ }^{\prime}-\mathrm{IFR}_{E i}{ }^{\prime}\right) \sin \gamma_{i} \cos \gamma_{i}\right. \\
& \left.\cdot \frac{\int_{B}^{\beta_{i}} F\left(t, \phi_{i}\right) d t}{\left(\frac{D}{2}\right)^{2} \int_{0}^{2 \pi} \int_{0}^{1} F(t, \phi) t d t d \phi}\right\} \mid
\end{aligned}
$$

Clearly, if $\sin \gamma_{i} \cos \gamma_{i}$ is zero (e.g., principal-plane struts), cross polarization is not generated by the $i$ th strut. Other strut configurations, e.g., an equiangular tripod, will also not generate boresight cross polarization because of cancellation properties of the total geometry, although each strut individually may generate a cross polarized component.

Because of its relatively high radius of gyration, a square cross section strut is attractive from a mechanical point of view. Determination of its IFR values using (1a) dan (1b) 

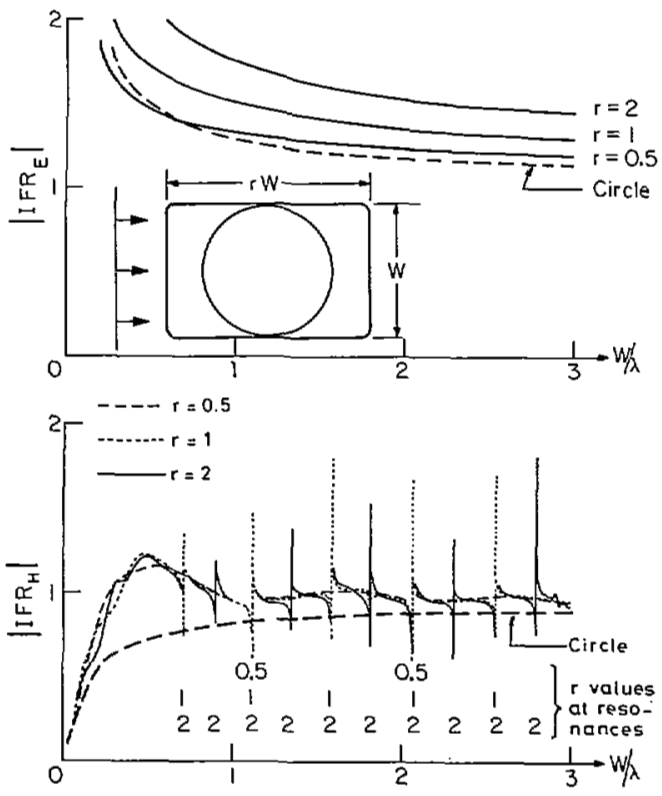

Fig. 3. IFR magnitudes for rectangular cylinders.

must be carried out using integral-equation techniques. However, standard moment-method techniques yield the IFR magnitudes plotted in Fig. 3. Internal resonances in the structure cause the numerical procedures to become unstable, yielding the remarkable, but spurious resonant peaks shown in the figure for $H$ polarization (corresponding to the TM waveguide frequencies at cutoff). Although these resonances also occur in the $E$ polarization current solutions, they are nonradiative components which do not cause erroneous scattered fields except in very narrow regions in which the matrix is too ill-conditioned to be accurately inverted. It is the purpose of this paper to present both numerical and experimental techniques whereby the IFR's of square, perfectly conducting cylinders can be correctly determined, and to discuss the consequences of the results on aperture blocking. (Although the paper is restricted to perfect conductors, the IFR method can also be applied to dielectric struts.)

\section{Numerical TeChNQues for Evaluating the IFR of a Rectangular Cylinder}

The problem of scattering by rectangular cylinders has been discussed by several authors [5]-[7]. However, as mentioned in the introduction, the standard method-ofmoments solution exhibited serious instabilities at frequencies associated with internal resonances. In a previous account [8] it was shown how the condition number could be used to detect these unwanted resonances and evaluate various methods used for removing their effect. This section of this paper will discuss how internal constraints can be used in the method-of-moment calculations to obtain correct values for IFR at the resonant frequencies.

The results for each polarization are usually calculated using different formulations. The usual formulation for the $E$-wave polarization is the following $E$-field integral equation which enforces the condition that the total tan-

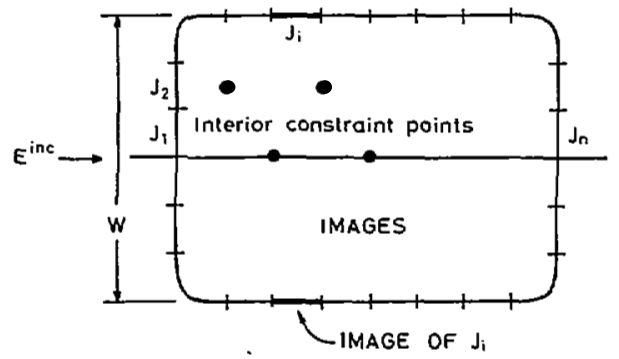

Fig. 4. Cross section of rectangle showing segmentation and position of internal constraint points.

gential $E$-field be zero along the surface of the cylinder:

$$
\frac{k Z_{0}}{4} \int_{S_{1}} H_{0}^{(2)}\left(k\left|\rho-\rho_{S}\right|\right) J_{S z}(\rho) d l=E_{z}^{\text {inc }}\left(\rho_{S}\right) .
$$

After the current has been determined, IFR $_{E}$ can be calculated by (la). For the $H$-wave polarization an $H$-field integral equation is often used. Recalling that $H_{z}$, the total $z$-directed $H$-field just external to the cylinder, is equal to $J_{S c}$, the circumferential current density, one can derive

$H_{z}\left(\rho_{S}\right)+\frac{j k}{4} \int_{S_{1}}(a \cdot n) H_{1}^{(2)}\left(k\left|\rho-\rho_{S}\right|\right) H_{z}(\rho) d l=H_{z}^{\text {inc }}\left(\rho_{S}\right)$.

Here $H_{1}{ }^{(2)}$ is the Hankel function of the first order, second kind, $\boldsymbol{n}$ is the outward normal vector from the cylinder, $\boldsymbol{a}$ is a unit vector pointing from the source point to the observation point, $\rho$ and $\rho_{S}$ are vectors to the source and observation points, respectively, and $\mathrm{IFR}_{H}$ can be calculated from $H_{z}$ by (1b).

For both polarizations the integral equations obtained can be easily discretized by the method-of-moments in the standard way. The discretization may be done by dividing the perimeter of the cross section into equal length segments and solving for currents $J_{i}$ that are uniform over these segments (see Fig. 4). Applying the technique of point matching, we will specify the value of the field at the midpoints of each segment. Since the incident wave has been restricted to be normal to the front face, one can use the resulting symmetry to half the order of the matrix while keeping the same number of segments. Applying the symmetry consideration, therefore, is equivalent to using pairs of rectangular currents as the expansion functions. In calculating the matrix elements, one must use a small argument expansion of the kernel for the self elements; for all the other elements, the approximation that all the current over a segment is concentrated at its midpoint is usually sufficient. An easy way to improve this last approximation is to calculate the fields at the testing point due to currents at the midpoint and the two endpoints of the source segment weighted by the proper Simpson rule coefficients.

However, when the method of moments is applied in the usual way, resonances can produce erroneous results at certain frequencies which correspond to waveguide modes at cutoff in the interior of the cylinder. First one should note that assumed symmetry of the problem eliminates any potentially troublesome asymmetric resonances. The way 
to eliminate all interior resonances is the use of extended boundary conditions [9]. Since one can fill the interior region of the cylinder with a perfect electric conductor without changing currents or scattered fields, restricting the total field to zero at specified interior points is a valid condition for the fields to satisfy. The matrix equation is modified by using these internal points as additional testing points in the method of moments. The resulting overdetermined matrix equation can be solved by multiplying both sides of the equation by the transpose-conjugate of the matrix yielding a new equation with a square matrix. In a formal sense this is applying the Moore-Penrose pseudoinverse for which theory guarantees the minimumnorm least squares solution [10]. Varying the number of internal points from one to six does not significantly change results. For the distribution of interior points it is important that they are not all on the nodal lines of a modal field or else the problem will be as ill-conditioned as before. The four interior points used for calculations in this paper (see Fig. 4) were chosen to be as independent as possible.

\section{EXPerimentally Determíned IFR's for SQuare CYlinders}

\section{A. Theory of Experiment}

Parallel plate waveguides have frequently been used to measure two-dimensional scattering properties. However, these measurements are difficult to carry out for the $H$ polarization. Consequently a three-dimensional scattering range was used for the measurements of this paper (Fig. 5(a)). $H_{1}$ is an $S$ band transmitting horn, and $H_{2}$ is an $S$ band receiving horn.

Neglecting currents on the top and bottom of the cylinder

$$
\boldsymbol{E}_{\mathrm{scat}}\left(0, \eta_{0}, 0\right)=-\frac{j \omega \mu}{4 \pi} \int_{-L / 2}^{+L / 2} \oint_{S_{\mathrm{l}}}\left(J_{S} \cdot \bar{\Gamma}\right) d l d z
$$

where, in terms of Fig. 5(a),

$$
\begin{aligned}
\rho & =\sqrt{\xi^{2}+\left(\eta_{0}^{\prime}+\eta\right)^{2}+z^{2}}=\sqrt{\rho_{0}^{2}+z^{2}} \\
r & =\sqrt{\xi^{2}+\left(\eta_{0}-\eta\right)^{2}+z^{2}}=\sqrt{r_{0}^{2}+z^{2}} \\
\rho_{0} & =\sqrt{\xi^{2}+\left(\eta_{0}^{\prime}+\eta\right)^{2}} \\
r_{0} & =\sqrt{\xi^{2}+\left(\eta_{0}-\eta\right)^{2}} .
\end{aligned}
$$

Now define

$$
J_{S} \cdot \bar{\Gamma}=\left(\bar{J}_{S} \cdot \bar{\Gamma}\right)^{\prime} \frac{e^{-j k \rho}}{\rho} \frac{e^{-j k r}}{r}
$$

where the phase of $\left(J_{S} \cdot \bar{\Gamma}\right)^{\prime}$ varies only very slowly in $z$. Evaluating the $z$-integral using saddle-point techniques [11] yields (assuming the cylinders to be long in terms of wavelengths and the horn illumination of the ends to be relatively low)

$$
\begin{aligned}
& \boldsymbol{E}_{\text {seat }}\left(0, \eta_{0}, 0\right) \\
& =-\frac{j \omega \mu}{4 \pi} e^{-j \pi / 4} \sqrt{\frac{2 \pi}{k}} \int_{S_{1}}\left(J_{S} \cdot \bar{\Gamma}\right)_{0}^{\prime} \frac{e^{-j k\left(\rho \div r_{0}\right)}}{\sqrt{\rho_{0} r_{0}\left(\rho_{0}+r_{0}\right)}} d l
\end{aligned}
$$

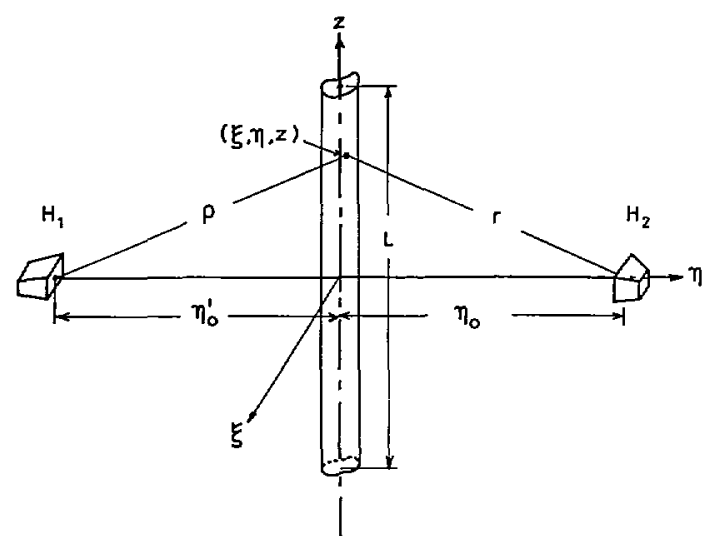

(a)

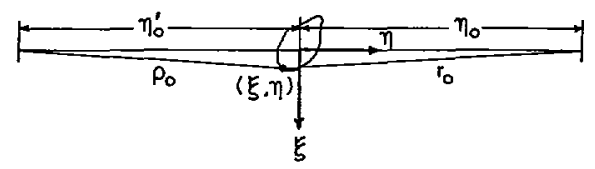

(b)

Fig. 5. (a) Geornetry of two-horn forward-scattering range. (b) Cross section geometry.

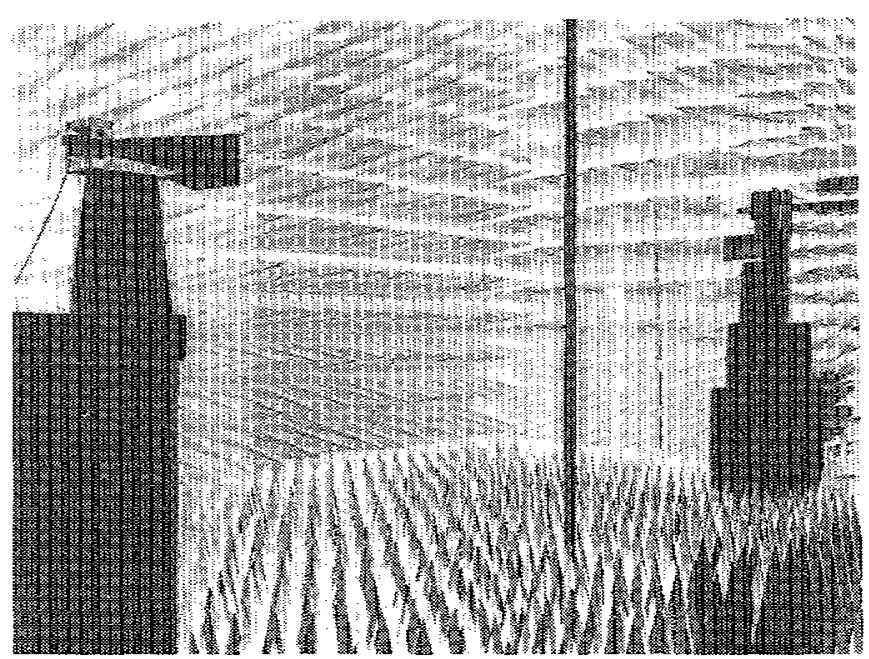

Fig. 6. Forward-scattering range in anechoic chamber of Technical University of Denmark.

where $\left(\boldsymbol{J}_{S} \cdot \bar{\Gamma}\right)_{0}^{\prime}=J_{S z} \boldsymbol{a}_{z} /\left(e^{-j k \rho_{0}} / \rho_{0}\right)$. If the cylinder is in the far-field of the horns and vice versa (with respect to maximum width) then the following approximations are justified in phase expressions (see Fig. 5(b)):

$$
\begin{aligned}
\rho_{0}+r_{0} & \cong \eta_{0}+\eta_{0}{ }^{\prime} \\
\rho_{0} & \cong \eta_{0}{ }^{\prime}+\eta
\end{aligned}
$$

and in amplitude expressions

$$
\begin{aligned}
& r_{0} \cong \eta_{0} \\
& \rho_{0}=\eta_{0}{ }^{\prime} .
\end{aligned}
$$

Then

$$
E_{\text {scat }}\left(0, \eta_{0}, 0\right) \cong \frac{e^{j \pi / 4} w a_{z} E_{0} e^{-j k\left(\eta_{0}+\eta_{0^{\prime}}\right)}}{\sqrt{2 \eta_{0} \eta_{0}{ }^{\prime}\left(\eta_{0}+\eta_{0}{ }^{\prime}\right)}} \operatorname{IFR}_{E}
$$




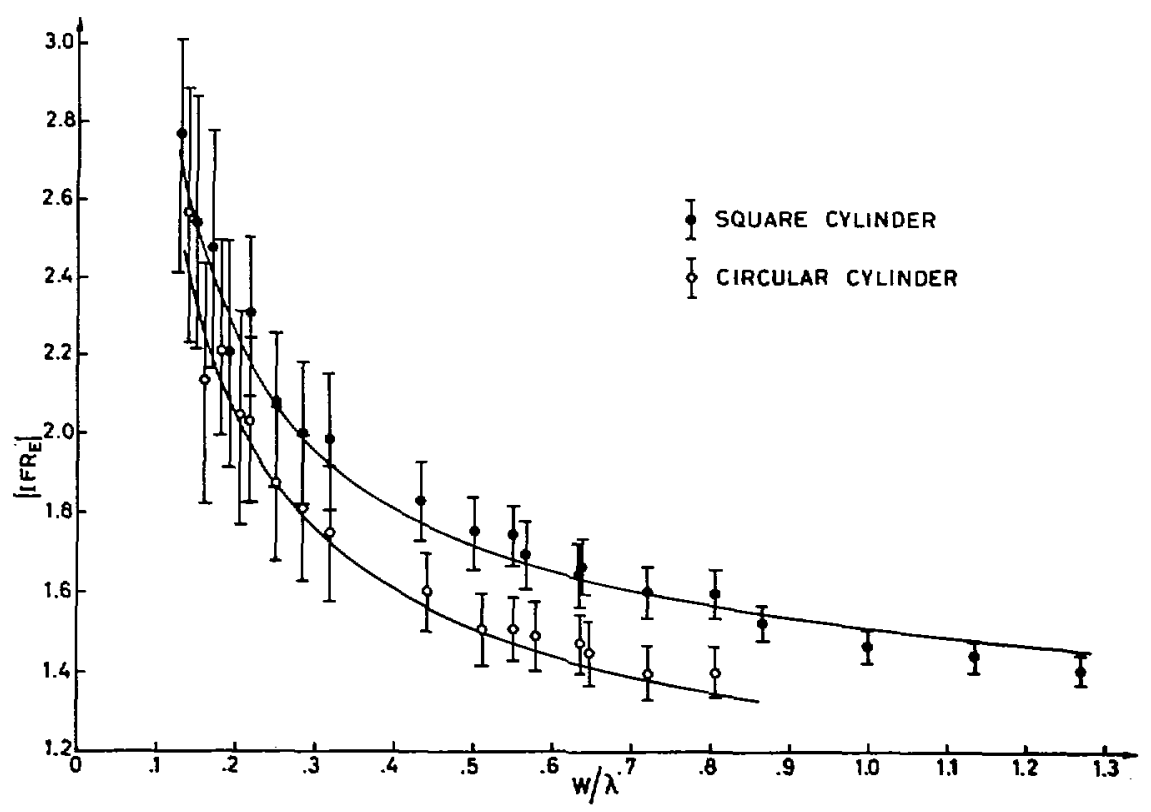

Fig. 7. IFR $_{E}$ magnitude for square and circular cylinders.

and the total field at $P$ in the presence of the cylinder is

$$
\begin{aligned}
& E_{\mathrm{total}}\left(0, \eta_{0}, 0\right) \\
& \quad=E_{\mathrm{inc}}\left(0, \eta_{0}, 0\right)\left[1+w e^{j(\pi / 4)} \operatorname{IFR}_{E} \sqrt{\frac{\eta_{0}+\eta_{0}{ }^{\prime}}{\lambda \eta_{0} \eta_{0}{ }^{\prime}}}\right]
\end{aligned}
$$

where

$$
E_{\mathrm{inc}}\left(0, \eta_{0}, 0\right)=\frac{E_{0}^{\prime} e^{-j k\left(\eta_{0} \div \eta_{0}{ }^{\prime}\right)}}{\eta_{0}+\eta_{0}^{\prime}} a_{z}
$$

is the incident field from $H_{1}$ to $H_{2}$ in the absence of the cylinder.

As described in the next section the amplitude change $\Delta \alpha$ and the phase change $\Delta \phi$ in the field incident on $\mathrm{H}_{2}$ is measured when the cylinder is inserted between $H_{1}$ and $H_{2}$. Thus, $E_{\text {inc }}\left(0, \eta_{0}, 0\right) e^{-\Delta \alpha} e^{-j \Delta \phi}$ denotes the total field $E_{\text {total }}\left(0, \eta_{0}, 0\right)$ which is then inverted to yield

$$
\mathrm{IFR}_{E}=\left(e^{-\Delta \alpha} e^{-j \Delta \phi}-1\right) \frac{e^{-j(\pi / 4)}}{w} \sqrt{\frac{\lambda \eta_{0} \eta_{0}^{\prime}}{\eta_{0}+\eta_{0}{ }^{\prime}}} .
$$

An identical expression, used in calculating experimentally determined IFR values, may be derived for the $H$ polarization.

\section{B. Experimental Setup}

The measurements were carried out in a radio anechoic chamber as shown in Fig. 6. The IFR was determined at $2.6,3.0,3.4$, and $3.8 \mathrm{GHz}$ for cylinders of square and circular cross section. In order to obtain nearly a decade variation in the ratio $w / \lambda$ from about 0.15 to 1.3 , the $\mathrm{IFR}_{E}$ and $\mathrm{IFR}_{H}$ were measured at the above frequencies for cylinders of widths of about $1.5,2.5,5,6.35$, and $10 \mathrm{~cm}$. Circular and square cylinders were used for all values of the width, except for $w=10 \mathrm{~cm}$, for which only a square cylinder was available. The length of all cylinders was about $6 \mathrm{~m}$. The distance between the apertures of the transmitting and receiving horns was $7.50 \mathrm{~m}$. The $3 \mathrm{~dB}$ beamwidths of the horns were $9^{\circ}$ in the $E$ - and $H$-planes, and the horn radiation was $20-30 \mathrm{~dB}$ below the peak level in the direction of the ends of the cylinder. A special support for the cylinders was arranged approximately halfway between the horns in such a manner that the different cylinders could be easily inserted and removed. Furthermore, the support was mounted on an automated rolling cart so that the influence of disturbing reflections between the cylinders and the horns could be reduced by averaging the recorded interference patterns.

The amplitude changes $\Delta \alpha$ and phase changes $\Delta \phi$ of the received signal due to insertion of cylinders between the horns were detected using a microwave bridge which included a transmission test unit and a network analyzer. The transmitted signal was frequency stabilized in order to obtain an accuracy of a few hundredths of a decibel in the measurements of amplitude changes and a few hundredths of a degree in the measurements of phase changes.

\section{Results}

The experimentally measured IFR $R_{E}$ magnitudes, based on (16) and the measured $\Delta \alpha$ and $\Delta \phi$ values, are compared in Fig. 7 with the theoretical values (solid curves) for both square and circular cross sections. The error flags are based on a peak uncertainty of $0.05 \mathrm{~dB}$ in $\Delta \alpha$ and $0.05^{\circ}$ in $\Delta \phi$. Furthermore, fixed values of the reflections from the walls, floor and ceiling of the chamber are taken into account. The larger error flags for small $w / \lambda$ values reflect the larger percentage effect these fixed uncertainties have for small $\Delta \alpha$ and $\Delta \phi$. Since $\Delta \alpha$ values were generally of the order of $2 \mathrm{~dB}$ or less and $\Delta \phi$ values were less than $10^{\circ}-12^{\circ}$, the factor $e^{-\Delta x} e^{-j \Delta \phi}$ in (16) was generally of the order of unity in magnitude, and subtracting unity in calculating the IFR 


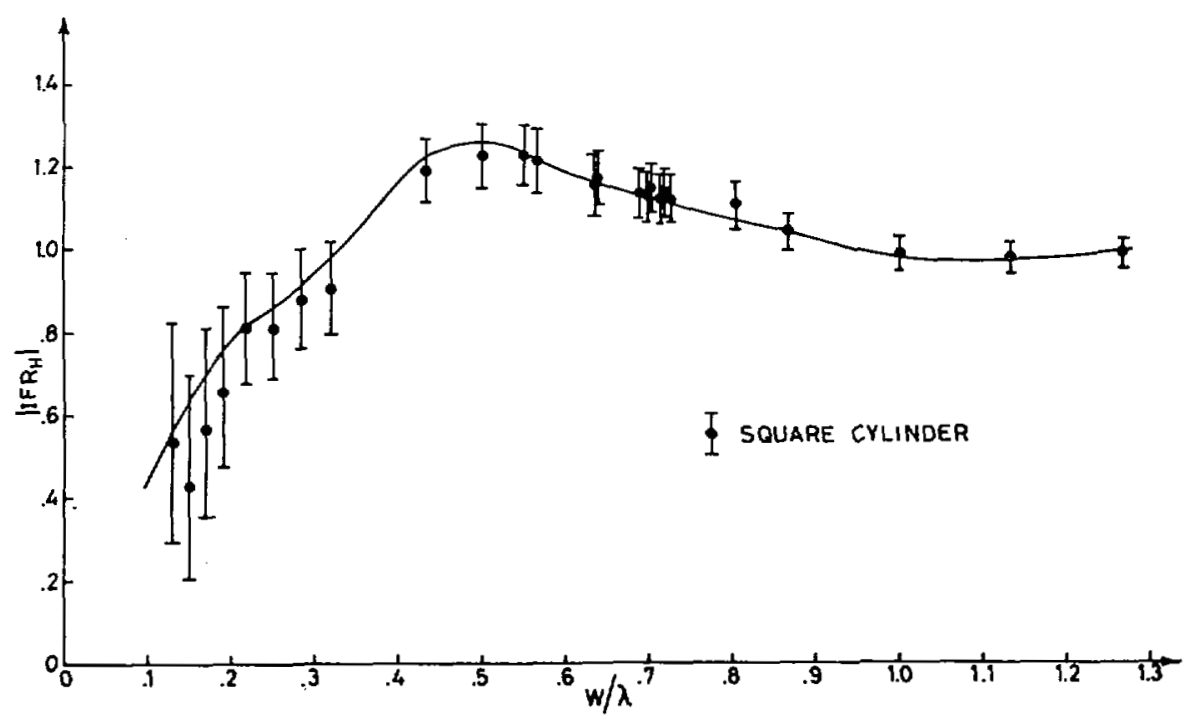

(a)

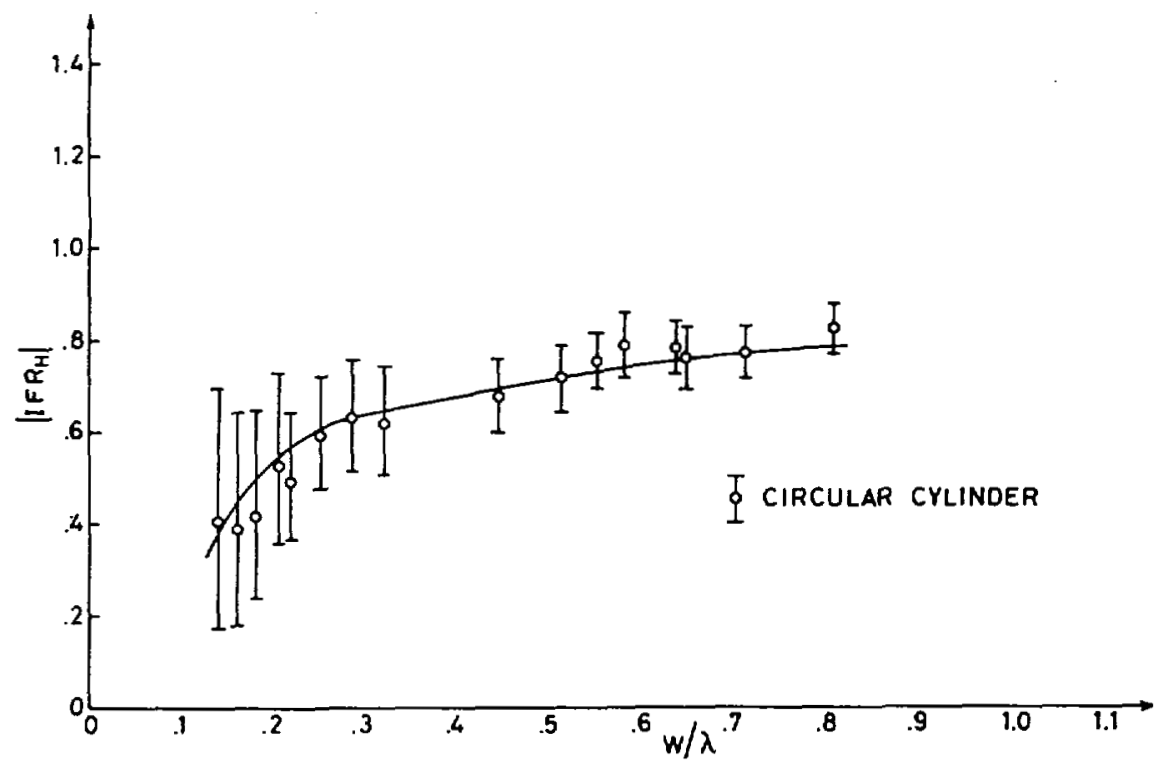

(b)

Fig. 8. (a) IFR ${ }_{H}$ magnitude for square cylinder. (b) $\operatorname{IFR}_{H}$ magnitude for circular cylinder.

generally magnified any experimental uncertainties. Thus the measurements were extremely sensitive to any random or systematic errors. Finally, since a moving cart was used for the measurements, the 1.5 - and $2.5-\mathrm{cm}$ diameter cylinders were subject to considerable mechanical oscillation (swinging), which may have caused enhanced data scatter for small $w / \lambda$.

With a few exceptions, the experimental error flags may be seen to overlap the calculated $E$-wave IFR magnitudes both for the square (based on moment-method analysis) and circle (based on (2a)). Both the experimental and theoretical results for the circular cross section are consistently 10-15 percent less than those for the square, indicating that the magnitude of the forward scattered field for $E$-wave incidence is less for a circular cylinder than for a square of the same width.

The experimentally measured $\mathrm{IFR}_{H}$ magnitudes are compared in Fig. 8(a) with the theoretical curve for square cross section, and in Fig. 8(b) for circular cross section. The experimental error flags generally overlap the theoretical curves. There is no evidence of the spurious resonances in Fig. 3 either in the theoretical curve or in the experimental data (as seen, for example, by the five points surrounding $w / \lambda=\sqrt{2}$ in Fig. 8(a), a resonance of the "internal" structure). Again, the $H$-wave IFR magnitudes for the square cross section are consistently greater than those for the circular. The "hump" in the curve for the square cross section is sufficiently pronounced that at $w / \lambda=0.5$, for example, the IFR is nearly 50 percent greater than the value for a circular cylinder of the same width.

Experimentally measured IFR phase data was consistently $8^{\circ}-12^{\circ}$ in delay of the corresponding theoretical values for both cross sections, both polarizations, and all cylinder sizes and frequencies. It was not known whether this 


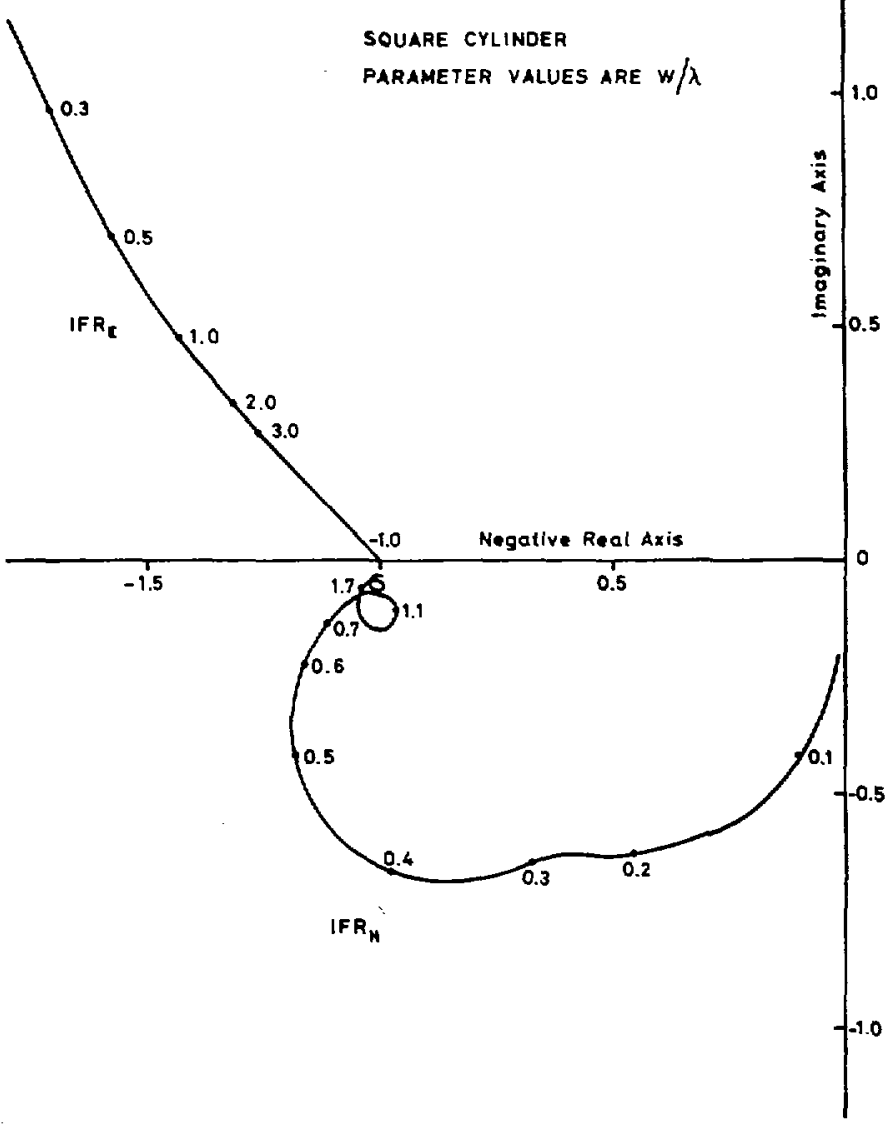

Fig. 9. Complex $\mathrm{IFR}_{E}$ and $\mathrm{IFR}_{H}$ for square cylinder.

consistent discrepancy was due to the measurement procedures or approximations in the theory. However, the significance of this small discrepancy is quantitatively very little in its effects on aperture blocking.

\section{Discussion of the Results for Square Cylinders}

The IFR's for a square cylinder are plotted on the complex plane in Fig. 9. This figure resembles Fig. 2 for the circular cylinder except for its more complicated structure and enhanced magnitude for the $H$ polarization. Similarly a table of IFR values for $H$ polarization is given in Table I for rectangular cylinders with face-on incidence. The depth-width ratio $r$ is shown in Fig. 3.

The enhanced IFR magnitudes for rectangular and square cylinders have significant implications on aperture blocking losses and boresight cross polarization levels as defined in (4) and (5). In particular, the "super-enhanced" IFR magnitude may cause greatly increased blocking by $H$-plane struts for a linearly polarized antenna, or by any strut for a circularly polarized antenna. The question is considerably complicated, however, by the more favorable radius of gyration of a square strut relative to a circular one, so that a square strut of smaller width will achieve the same mechanical properties as a somewhat larger circular strut.
TABLE I

COMplex IFR R $_{H}$ Magntude AND Phase for Rectangular CYLINDERS

\begin{tabular}{|c|c|c|c|c|c|c|}
\hline & \multicolumn{2}{|c|}{$x=\frac{2}{2}$} & \multicolumn{2}{|c|}{$x=1$} & \multicolumn{2}{|c|}{$r=2$} \\
\hline$v / \lambda$ & Mag. & Phase & Mag. & Pbase & Mag. & Phase \\
\hline .05 & .1800 & -92.27 & .2028 & -93.59 & .2299 . & -98.22 \\
\hline . & . .3873 & -97.74 & .4303 & -103.22 & .4382 & -113.20 \\
\hline .15 & .6154 & -106.56 & .6382 & -115.30 & .5483 & -121.36 \\
\hline .2 & .8315 & -117.55 & .7784 & -126.17 & .6772 & -120.16 \\
\hline .25 & .1942 & -128.89 & .8589 & -133.10 & .9101 & -125.22 \\
\hline .3 & 1.0876 & -138.78 & .9375 & -136.38 & 1.0628 & -137.07 \\
\hline .4 & 3.1500 & -151.85 & 1.1842 & -145.81 & 1.1321 & -148.49 \\
\hline .5 & 2.1818 & -159.86 & 1.2550 & -160.60 & 1.2374 & -160.59 \\
\hline .6 & 1.1821 & -167.09 & 1.1834 & -168.96 & 1.1711 & -168.17 \\
\hline .7 & 1.1327 & -172.44 & 1.1204 & -172.99 & 1.1296 & -172.79 \\
\hline .8 & 1.0688 & -175.17 & 1.0660 & -175.27 & 1.0637 & -175.43 \\
\hline .9 & 1.0140 & -175.83 & 1.0147 & -175.89 & 1.0128 & -176.01 \\
\hline 1.0 & .9763 & -175.04 & .9780 & -175.07 & .9779 & -175.14 \\
\hline 1.1 & .9639 & -173.40 & .9672 & -173.56 & .9624 & -173.93 \\
\hline 1.2 & .9800 & -172.06 & .9749 & -172.47 & .9701 & -172.06 \\
\hline 1.3 & 1.0087 & -171.91 & .9976 & -171.53 & 1.0016 & -171.75 \\
\hline 1.4 & 1.0264 & -172.61 & 1.0315 & -171.82 & 1.0240 & -172.25 \\
\hline 1.5 & 1.0435 & -273.70 & 1.0549 & -173.66 & 1.0496 & -173.69 \\
\hline 1.6 & 1.0497 & -175.14 & 1.0501 & -175.46 & 1.0487 & -175.42 \\
\hline 1.7 & 1.0422 & -176.43 & 1.0390 & -176.60 & 1.0394 & -176.50 \\
\hline 1.8 & 1.0233 & -177.24 & 1.0225 & -177.29 & 1.0224 & -177.30 \\
\hline 1.9 & 1.0064 & -177.42 & 1.0061 & -377.46 & 1.0049 & -177.51 \\
\hline 2.0 & .9932 & -177.08 & .9921 & -177.20 & .9926 & -177.15 \\
\hline 2.2 & .9939 & -175.85 & 9912 & -176.03 & .9905 & -175.84 \\
\hline 2.4 & 1.0136 & -175.88 & 1.0146 & -175.61 & 1.0156 & -175.71 \\
\hline 2.6 & 1.0244 & -177.03 & 1.0245 & -177.04 & 1.0276 & -177.09 \\
\hline 2.8 & 1.0132 & $-178.0 T$ & 1.0119 & -178.08 & 1.0157 & -178.05 \\
\hline 3.0 & .9966 & -177.99 & .9966 & -178.05 & .9998 & -277.91 \\
\hline
\end{tabular}

For example, the IFR's of a square cylinder of $0.608 \lambda$ width for normal face-on incidence are tabulated in Table II together with the corresponding IFR's for four reference cylinders of circular cross section. The reference cylinders are:

a) equal diameter, i.e., $0.680 \lambda$, which can be inscribed in the square cylinder;

b) equal cross sectional area, i.e., 0.461 square wavelengths (diameter $=0.766 \lambda$ );

c) equal circumference, i.e., $2.65 \lambda$ (diameter $=0.846 \lambda$ );

d) circular cylinder of $0.931 \lambda$ width, which can be circumscribed around the square cylinder.

The IFR magnitudes for electric, magnetic, and circular polarization are tabulated. In [2] it is shown that the IFR for circular polarization is equal to the algebraic mean of the $E$ - and $H$-wave IFR's. In order to compare the different 
TABLE II

Comparison of IFR's for Square Cylinders and Reference Circular Cylnnders-Normal Incidence

\begin{tabular}{|c|c|c|c|c|c|c|c|}
\hline & $\begin{array}{c}\text { vidth, } \\
\text { (wavelength) }\end{array}$ & $\left|\mathrm{IFR}_{\mathrm{E}}\right|$ & $\left|\operatorname{IFR}_{\mathrm{B}}\right|$ & $\left|\mathrm{IFR}_{\mathrm{CP}}\right|$ & $n\left|\operatorname{IFR}_{E}\right|$ & $\otimes\left|I F R_{H}\right|$ & $v\left|\mathrm{FR}_{\mathrm{CP}}\right|$ \\
\hline $\begin{array}{l}\text { Square } \\
\text { cylinder }\end{array}$ & 0.680 & 1.612 & 1.099 & 1.314 & 1.096 & 0.747 & 0.893 \\
\hline $\begin{array}{l}\text { Circular } \\
\text { inscribed }\end{array}$ & 0.680 & 1.397 & 0.761 & 0.998 & 0.950 & 0.517 & 0.679 \\
\hline $\begin{array}{l}\text { Circular } \\
\text { equal } \\
\text { cross-section }\end{array}$ & 0.766 & 1.362 & 0.777 & 0.994 & 1.044 & 0.595 & 0.762 \\
\hline $\begin{array}{l}\text { Circular } \\
\text { equal } \\
\text { circumference }\end{array}$ & 0.846 & 1.336 & 0.794 & 1.000 & 1.129 & 0.671 & 0.845 \\
\hline $\begin{array}{l}\text { Circular } \\
\text { circumscribed }\end{array}$ & 0.931 & 1.312 & 0.805 & 1.000 & 1.221 & 0.749 & 0.931 \\
\hline
\end{tabular}

geometries the quantity (width) $*$ (IFR) is also tabulated. This quantity yields the effective blocking contribution to the forward scattered field since the IFR alone is normalized by the width.

The blocking parameters of the four reference cylinders are plotted in the four bottom rows of Table II. The electric IFR magnitudes decrease toward unity as the diameter increases while the magnetic IFR magnitudes increase toward unity. The circular polarization magnitudes are close to unity over the range. The $w$ IFR product for the two smaller circular cylinders is less than that of the square cylinder for the $E$-wave and circular polarization. Because the magnetic scattering appears enhanced for the square cylinder, three of the circular cylinders have smaller wIFR products than the square cylinder. A complete comparison of the circular versus square cylinders involves both the RF and mechanical properties of interest. For example, if equal cross sectional areas are desired (equal support strength) the circular cylinders have 5 percent less $E$-wave blocking, 20 percent less $H$-wave blocking, and 15 percent less blocking for circular polarization. While no definitive figure of merit can be easily formulated for a comparison of the two cross sectional geometries, it may be concluded that the data from Table $I$ and Figs. 7 and 8 provide a quantitative means by which square cylinders can be selected for a particular strut support application.

\section{ACKNOWLEDGMENT}

The authors wish to thank P. Laugesen and N. O. Nielsen who performed most of the measurement work and mechanical arrangements.

\section{REFERENCES}

[1] A. F. Kay, "Electrical design of metal space frame radomes," IEEE Trans. Antennas Propagat., vol. AP-13, pp. 188-202, Mar. 1965.

[2] W. V. T. Rusch, "Application of two-dimensional integralequation theory to reflector-antenna analysis," TM 33-478, Jet Propulsion Laboratory, Pasadena, Calif., May 15, 1971.

[3] J. Ruze, "Feed support blockage loss in parabolic antennas," Microwave J., vol. 11, no. 12, pp. 76-80, 1968.

[4] W. V. T. Rusch, "Analysis of blockage effects on gain and sidelobes by struts of arbitrary cross-section in a dual-reflector antenna," in 1971 G-AP Int. Symp. Digest, UCLA, Los Angeles, Calif., Sept. 22-24, 1971, p. 211.

[5] R. F. Harrington, Field Computation by Moment Methods. New York: Macmillan, 1968, pp. 41-61.

[6] K. Mei and J. G. Van Bladel, "Scattering by perfectly conducting rectangular cylinders," IEEE Trans. Antennas Propagat., vol. AP-11, pp. 185-192, Mar. 1963.

[7] J. C. Bolomey and W. Tabbara, "Numerical aspects on coupling between complementary boundary value problems," IEEE Trans. Antennas Propagat., vol. AP-21, pp. 356-363, May 1973.

[8] C. Klein and R. Mittra, "An application of the 'condition number' concept to the solution of scattering problems in the presence of the interior resonant frequencies," submitted to IEEE Trans. Antennas Propagat.

[9] P. C. Waterman, "Matrix formulation of electromagnetic scattering," Proc. IEEE, vol. 53, pp. 805-812, Aug. 1965.

[10] C. R. Rao and S. K. Mitra, Generalized Inverse of Matrices and Its Applications. New York: Wiley, 1971.

[11] M. G. Andreasen and A. F. Kay, "Scattering from a metallic cylinder of arbitrary cross-section," TRG FR-220-2, Nov. 9, 1962. 